\title{
FUNÇÃO ALOMÉTRICA DE BIOMASSA COM IMAGENS DE SATÉLITE DE ALTA RESOLUÇÃO ESPACIAL
}

\section{BIOMASS ALLOMETRIC FUNCTION WITH SATELLITE IMAGES OF HIGH SPATIAL RESOLUTION}

\begin{abstract}
Fabrício Lopes de Macedo ${ }^{1}$ Adélia Maria de Oliveira Sousa ${ }^{2}$ Ana Cristina Gonçalves ${ }^{3}$ Hélio Ricardo Silva ${ }^{4}$ Ricardo Antonio Ferreira Rodrigues ${ }^{5}$
\end{abstract}

\section{RESUMO}

O presente estudo teve como objetivo ajustar funções para estimar por meio de dados derivados de imagens de satélite de alta resolução espacial, a biomassa total de Eucalyptus em escala local e regional. Para este fim, foram ajustados modelos, combinando os valores de biomassa estimada por meio de parcelas do inventário florestal associadas com índices de vegetação (IV) baseados em imagem do satélite Pléiades. A função com que apresentou melhor performance na estimativa da biomassa total, foi aquela que utilizou como variável independente o IV-SAVI, apresentando um coeficiente de determinação $\left(\mathrm{R}^{2}\right)$ de $64,6 \%$, no entanto, sem grande diferença para o NDVI e SR. As funções ajustadas poderão ser utilizadas em regiões que apresentem as mesmas espécies, clima e propriedades locais parecidas com as do presente estudo. Esta abordagem pode ser usada como uma ferramenta de baixo custo para produzir estimativas de biomassa em escala local e regional.

Palavras-chave: Eucalipto; Pléiades; índices de vegetação.

\begin{abstract}
The aim of this study was to fit functions to estimate with data derived from high spatial resolution satellite images, the total biomass of Eucalyptus at local and regional scale. For this purpose, models were fit combining values of biomass estimated from forest inventory plots and vegetation indices based on Pléiades images. The function with which presented the best performance in the estimation of the total biomass, was the one that used as an independent variable the IV-SAVI, presenting a coefficient of determination $\left(\mathrm{R}^{2}\right)$ of $64.6 \%$, however without great difference for the NDVI and SR. The fit functions can be used in other regions for the same species, and similar climate and local characteristics. This approach can be used as a low cost tool to produce estimations of biomass at local and regional level.
\end{abstract}

Keywords: Eucalyptus; pléiades; vegetation indices.

1 Engenheiro Agrônomo, Dr., Pesquisador do Centro de Investigação e de Tecnologias Agroambientais e Biológicas, Universidade de Trás-os-Montes e Alto Douro, Quinta de Prados, 5000-801, Vila Real, Portugal. fabriciolmacedo@ hotmail.com

2 Engenheira Geográfica, Dra ${ }^{\mathrm{a}}$, Professora Auxiliar do Departamento de Engenharia Rural, Escola de Ciências e Tecnologia, Instituto de Ciências Agrárias e Ambientais Mediterrânicas, Universidade de Évora, Apartado 94, 7002 - 554 Évora, Portugal. asousa@uevora.pt

3 Engenheira Silvicultora, $\mathrm{Dr}^{\mathrm{a}}$., Professora Auxiliar com Agregação do Departamento de Engenharia Rural, Instituto de Ciências e Tecnologia, Instituto de Ciências Agrárias e Ambientais Mediterrânicas, Universidade de Évora, Apartado 94, 7002 - 554 Évora, Portugal. acag@uevora.pt

4 Engenheiro Agrônomo, Dr., Professor Assistente do Departamento de Fitossanidade, Engenharia Rural e Solos, Universidade Estadual Paulista "Júlio de Mesquita Filho" (Unesp - Ilha Solteira), Av. Brasil, 56, CEP 15385-000, Ilha Solteira (SP), Brasil. hrsilva@agr.feis.unesp.br

5 Engenheiro Agrícola, Dr., Professor Livre Docente do Departamento de Fitossanidade, Engenharia Rural e Solos, Universidade Estadual Paulista "Júlio de Mesquita Filho" (Unesp - Ilha Solteira), Av. Brasil, 56, CEP 15385-000, Ilha Solteira (SP), Brasil. ricardo@agr.feis.unesp.br

Recebido para publicação em 16/12/2015 e aceito em 20/06/2017

Ci. Fl., v. 28, n. 3, jul. - set., 2018 


\section{INTRODUÇÃO}

Os aspectos que abrangem a dinâmica florestal, seu desenvolvimento, sua importância e exploração sustentáveis, favoreceram o aumento de pesquisas nas últimas décadas. O foco principal dessa temática incide no fato de que, as florestas desempenham elevada influência no balanço dos gases do efeito estufa e em suas implicações para as mudanças climáticas do planeta (VISMARA, 2009). As florestas têm grande relevância na mitigação dos efeitos da concentração de $\mathrm{CO}_{2}$ na atmosfera, dado que o gás é o principal responsável pelo efeito estufa segundo o IPCC (INTERGOVERNMENTAL PANEL ON CLIMATE CHANGE, 2001). Matheus (2012) comenta que a ideia principal associada ao conceito de sequestro de carbono consiste na preservação da vegetação nativa e/ou no reflorestamento para que, através da fotossíntese, o $\mathrm{CO}_{2}$ fosse absorvido e/ou mantivesse o estoque de carbono nas plantas (YU, 2004).

Segundo Renner (2004), o sequestro de carbono florestal é uma das principais alternativas para mitigar a situação referente ao processo de elevação da temperatura do planeta. Fearnside e Guimarães (1996) salientam que de maneira contrária às plantas de curto ciclo de vida, as árvores com ciclo de vida longo acumulam carbono e biomassa. Para Higuchi et al. (1998), é importante a realização da estimação de biomassa florestal, já que, ela se relaciona diretamente com os estoques de carbono, que são utilizados na quantificação de $\mathrm{CO}_{2}$ liberado na atmosfera durante o desenvolvimento da decomposição. Segundo Sanquetta (2002), quanto mais preciso for o método de estimação da biomassa, maior será a consistência da quantificação de carbono estocado e/ou liberado na atmosfera pelos ecossistemas florestais.

As principais metodologias utilizadas na estimativa da biomassa florestal podem ser separadas em duas categorias: método direto (ou destrutivo) e o método indireto (não destrutivo). No primeiro método, as árvores são derrubadas, seus componentes são separados e então é determinado o peso seco; esse método é considerado muito preciso e caro (NUNES, 2011). No segundo método, as metodologias têm como base os dados provenientes dos inventários florestais, em que, com o auxílio de funções alométricas, transformam a altura total, o diâmetro e/ou volume em estimativas de biomassa (SOMOGYI et al., 2006).

Watzlawick e Kirchner (2004) afirmam que os avanços tecnológicos atuais, ligados às imagens provenientes dos sensores remotos e o desenvolvimento do processamento das imagens, têm possibilitado uma melhor caracterização da estrutura florestal e, consequentemente, maior facilidade na estimação da biomassa e do carbono estocado. Segundo Rosenqvist et al. (2003), essas estimativas têm como fundamento básico a combinação de elementos de radiância e/ou reflectância, provenientes das imagens de satélite, com informações obtidas no campo. Os alvos nas imagens de satélite normalmente são distinguidos através da reflectância em cada uma das bandas espectrais ou por meio de índices. Ponzoni e Shimabukuru (2009) afirmam que para o estudo de vegetação, as transformações radiométricas denominadas de índices de vegetação normalmente são muito utilizadas. Para Watzlawick et al. (2002), os dados provenientes de imagens de satélite são muito importantes na estimação de carbono e biomassa, tanto no cálculo de áreas, como na caracterização das estruturas florestais.

O objetivo do trabalho foi ajustar funções no processo da estimativa da biomassa total, por meio de dados derivados de sensoriamento remoto, especificamente com auxílio dos índices de vegetação. Obtendose assim funções para estimação da biomassa, sem que haja necessidade da realização de um inventário de campo, permitindo a monitorização ao longo do tempo e a redução dos custos.

\section{MATERIAL E MÉTODOS}

\section{Localidade de estudo}

O presente estudo, foi realizado na Fazenda Dois Irmãos do Buriti (Figura 1), assentada nas proximidades de Selvíria, localizada no estado do Mato Grosso do Sul. Segundo a categorização de Köppen, o clima da localidade de estudo pode ser classificado como sendo Aw, apresentando verão quente e chuvoso e inverno seco e ameno, apresentando médias anuais de precipitações na faixa de $1.370 \mathrm{~mm}$, concentrada entre março a outubro, apresentando ainda uma média anual de temperatura média na faixa $23,5^{\circ} \mathrm{C}$ (GARCIA et al., 2014). As classes de solos encontradas na presente na localidade de estudo, compreende os Argissolos Vermelhos e Latossolos Vermelhos (EMBRAPA, 1999). As principais tipologias florestais presentes na área 
são compostas essencialmente de eucalipto, e alguns pontos com Cerradão e Cerrado.
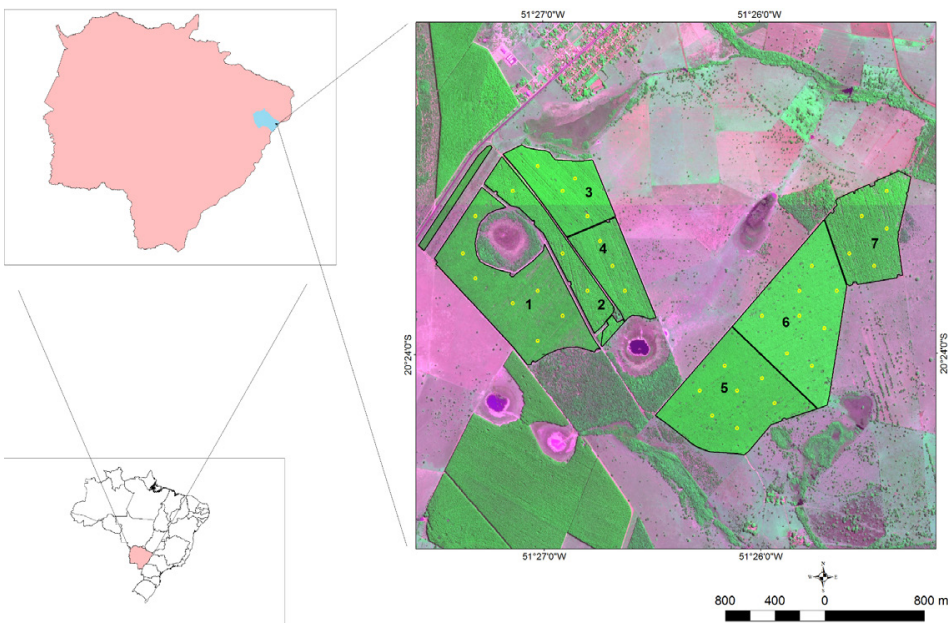

FIGURA 1: Parcelas de estudo (R3G4B2).

FIGURE 1: Plots of study (R3G4B2).

\section{Dados de satélite}

A imagem utilizada teve como data o dia 27 de Julho de 2013, e a mesma é oriunda do satélite Pléiades concedido na forma "Pan-sharpened" com a resolução espacial de $0,50 \mathrm{~m}$, apresentando quatro bandas multiespectrais, b1 - Azul (A) (430 - $550 \mathrm{~nm})$, b2 - Verde (500 - $620 \mathrm{~nm})$, b3 - Vermelho (V) (590 $710 \mathrm{~nm}$ ) e b4 - Infravermelho próximo (IVP) $(740$ - $940 \mathrm{~nm}$ ), georreferenciada e corrigida geometricamente pela empresa.

\section{Estimativa da biomassa das áreas de eucalipto}

Nos 7 talhões foram plantadas três espécies de eucalipto (Tabela 1) abrangendo 37 parcelas.

TABELA 1: Espécies de eucalipto por talhão.

TABLE 1: Eucalyptus species per compartment.

\begin{tabular}{cccc}
\hline Talhão & Espécie & Plantio & Dimensão do talhão/ha \\
\hline 1 & Eucalyptus urophylla & $10 / 20 / 2011$ & 70,82 \\
2 & Eucalyptus urophylla & $10 / 19 / 2011$ & 27,55 \\
3 & Eucalyptus urophylla x grandis & $5 / 9 / 2011$ & 33,97 \\
4 & Eucalyptus urophylla & $10 / 20 / 2011$ & 26,61 \\
5 & Eucalyptus urophylla x grandis & $5 / 4 / 2011$ & 67,8 \\
6 & Eucalyptus grandis & $5 / 2 / 2011$ & 72,25 \\
7 & & $10 / 24 / 2011$ & 42,19 \\
\hline
\end{tabular}


Foi realizado um inventário florestal quando as plantas alcançaram dois anos de idade, utilizando para tanto o "Método de área fixa com parcelas circulares", de $400 \mathrm{~m}^{2}$ e com um raio de 11,24 $\mathrm{m}$. Medidas a altura total e circunferência a altura do peito. Neste estudo foram usadas as funções alométricas de Mello e Gonçalves (2008) desenvolvidas com base em povoações de Eucalyptus grandis com 9 anos de idade (equações 1 a 7, em que: BioFuste - Biomassa do Fuste, BioCasca - Biomassa da Casca, BioFolha Biomassa da Folha, BioGalho - Biomassa do Galho, BioFina - Biomassa Raiz Fina, BioGrossa - Biomassa da Raiz Grossa, Ln - logaritmo neperiano, DAP - Diâmetro a 1,3 m de altura, H - altura total). A biomassa total é definida com a soma das suas componentes (equações 1 a 6 ).

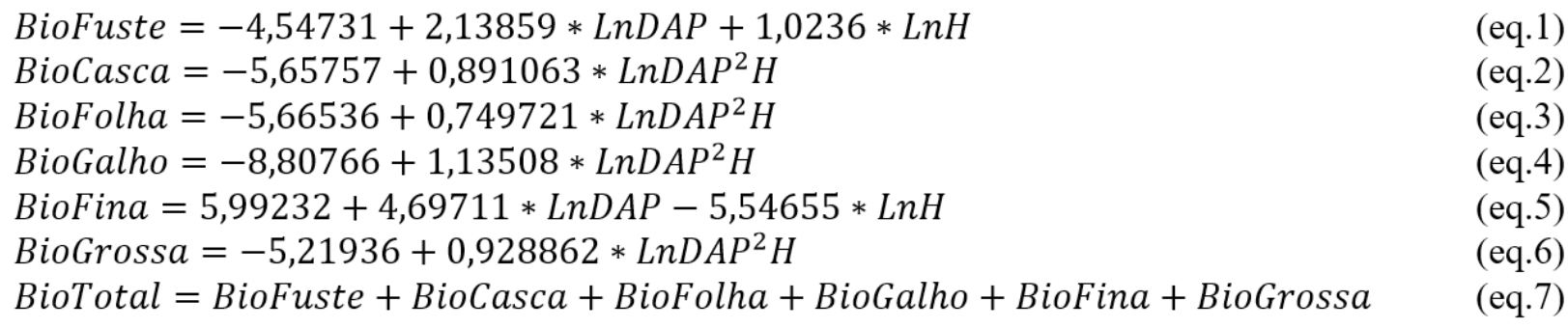

\section{Determinação dos IV (Índices de Vegetação)}

Utilizaram-se alguns IV, calculados basicamente com apoio das bandas específicas do satélite Pléiades, auxiliado pelo Spatial Analyst presente no ArcGIS (ENVIRONMENTAL SYSTEMS RESEARCH INSTITUTE, 2010). Os IV utilizados neste estudo foram a SR - Razão Simples (JORDAN, 1969):

$$
S R=\frac{I V P}{V}
$$

O NDVI - Índice de Vegetação por Diferença Normalizada (ROUSE et al., 1974):

$$
N D V I=\frac{I V P-V}{I V P+V}
$$

O SAVI - Índice de Vegetação Ajustado ao Solo (HUETE, 1988). O autor sugere que o valor de 1 é indicado para vegetação com baixa densidade, 0,5 é indicado para vegetação com densidade intermediária; 0,25 é indicado para vegetação densa.

$$
S A V I=\frac{I V P-V}{I V P+V+L} \times(L+1)
$$

E o Enhanced Vegetation Index (EVI) (HUETE; JUSTICE; VAN LEEUWEN, 1996; HUETE et al. 1997):

$$
E V I=2,5 \times \frac{I V P-V}{I V P+6 * V-7 * A+1}
$$

Em que: $\mathrm{IVP}=$ infravermelho próximo; $\mathrm{V}=$ Vermelho; $\mathrm{A}=\mathrm{Azul}$.

Todos os IV utilizaram a combinação de informações basicamente de duas ou três bandas espectrais, com intuito da melhoria do sinal da vegetação, reduzindo o efeito do solo, efeitos atmosféricos e de irradiância solares (JACKSON; HUETE, 1991). Foram calculadas as médias dos valores dos pixels dos IV relativos à parte central de cada parcela. 


\section{Desenvolvimento das funções alométricas e estimativa espacial da biomassa}

Foi efetuado o ensaio de correlação por meio do teste de Spearman, visto que a biomassa total não apresentava distribuição normal, avaliada pelo teste de normalidade de Shapiro Wilk (SHAPIRO; WILK; CHEN, 1968), em um nível de significância de 95\%. As funções de biomassa total foram ajustadas com procedimentos de regressão linear, pelo método dos mínimos quadrados (eq.12, onde é a constante, o declive da reta, Bio a biomassa total e IV é o índice de vegetação), proposto por diversos autores (RAUSTE; HÄME, 1994; DRAKE et al., 2002a; 2002b). As propriedades estatísticas das funções foram aferidas, segundo a sugestão de Sousa et al. (2015), pela soma dos quadrados dos resíduos (SQR), coeficiente de determinação $\left(\mathrm{R}^{2}\right)$ e coeficiente de determinação ajustado $\left(\mathrm{R}_{\mathrm{aj}}^{2}\right)$.

A soma dos quadrados dos resíduos estimados (PRESS, eq.13) e soma dos valores absolutos dos resíduos estimados (APRESS, eq.14), em que y é o valor observado, ŷ o valor estimado e i é o número da observação, $\mathrm{i}=1$...n, foram usados como um verdadeiro teste de validação, como proposto por Clutter et al. (1983) e Myers (1986). Com base nos critérios acima referidos, o melhor modelo será o que apresenta valores mais baixos de SQR, PRESS e APRESS, e os mais elevados $\mathrm{R}^{2}$ e $\mathrm{R}_{\text {aj }}^{2}$. A heteroscedasticidade ligada ao termo do erro dos modelos e a normalidade dos resíduos foi ponderada na forma gráfica, pela ligação entre os resíduos padronizados e a estimativa da variável dependente; nos gráficos de probabilidade normal e no teste de normalidade de Shapiro Wilk, para um nível de probabilidade de $99 \%$. A análise estatística foi realizada por meio do software R (R DEVELOPMENT CORE TEAM, 2012).

$$
\begin{aligned}
& \text { Bio }=\beta_{O}+\beta_{1} \times I V \\
& \text { PRESS }=\sum_{i=1}^{n}\left(y_{i}-\hat{y}_{i,-1}\right)^{2} \\
& \text { APRESS }=\sum_{i=1}^{n}\left|y_{i}-\hat{y}_{i,-1}\right|
\end{aligned}
$$

De modo a avaliar a distribuição espacial da biomassa total, a localidade de estudo foi dividida em uma malha de $20 \times 20 \mathrm{~m}$, representando os $400 \mathrm{~m}^{2}$ das parcelas, foi calculado o valor médio do SAVI (média aritmética de todos os pixels para cada quadrícula da malha), e a biomassa total por quadrícula com as funções alométricas da função M1. Foi gerado o mapa de biomassa total.

\section{RESULTADOS E DISCUSSÃo}

\section{Análise de correlação}

A matriz de correlação de Spearman apresentou intensas correlações negativas entre a biomassa total e todos os IV testados (Tabela 2), significando que, com a composição de bandas na faixa do espectro eletromagnético, especificamente, correspondente ao infravermelho próximo e ao vermelho que apresentam máximos valores de reflectância e forte absorção de luz respectivamente (WATZLAWICK; KIRCHNER; SANQUETTA, 2009). Os autores salientam ainda que, pode haver variações de resposta nas bandas citadas, devido à quantidade e tipo de pigmentos presentes na vegetação na faixa do vermelho e da interação da energia incidente no mesófilo com a quantidade de água na folha isso para a região do infravermelho.

O EVI apresentou os piores coeficientes de correlação. Os resultados das correlações obtidas destas pesquisas são análogas àquelas obtidas por Watzlawick, Kirchner e Sanquetta (2009) que analisaram a biomassa em plantios de Araucária angustifólia; Watzlawick, Koehler e Kirchner (2006) em plantios de Pinus taeda ambos com densidade muito alta. Os autores encontraram alta correlação negativa entre biomassa e NDVI (- 0,740 e - 0,800, respectivamente), SR (- 0,71 e - 0,750, respectivamente), e SAVI (- 0,77 e - 0,70, respectivamente). Maciel (2002) obteve correlações negativas de biomassa com o SAVI $(-0,83)$ usando imagens do satélite Landsat TM em uma floresta primária na Amazônia Oriental. Matos e Kirchner (2008) utilizando o satélite IKONOS II para estimar a biomassa de uma Floresta Ombrófila Densa, não obtiveram correlações entre o NDVI e o SAVI e a biomassa (- 0,070 e - 0,058, respectivamente). Neste estudo, a ligação entre os IV (SR, NDVI e SAVI) e a biomassa total, apresenta valores inferiores aos 
referidos por Watzlawick, Kirchner e Sanquetta (2009) e Maciel (2002). Tal resultado pode ser explicado por dois fatores: idade do plantio e espécie, visto que as funções disponíveis e utilizadas neste estudo são para plantações de Eucalyptus grandis com 9 anos de idade.

TABELA 2: Coeficientes de correlação entre a biomassa total e os índices de vegetação.

TABLE 2: Correlation coefficients between total biomass and the vegetation indices.

\begin{tabular}{c|ccccc}
\hline \multirow{4}{*}{ BIOTOTAL } & BIOTOTAL & NDVI & SR & SAVI & EVI \\
\cline { 2 - 6 } & 1 & & & & \\
NDVI & $-0,679$ & 1 & & & \\
SR & $-0,681$ & 0,998 & 1 & & \\
SAVI & $-0,672$ & 0,999 & 0,998 & 1 & \\
EVI & $-0,564$ & 0,811 & 0,818 & 0,818 & 1 \\
\hline
\end{tabular}

\section{Biomassa Total}

A função para a biomassa total com performance superior apresentou como variável independente o SAVI apresentando um coeficiente de determinação de 0,64. A função M4, da qual a variável independente é o EVI, apresentou o pior resultado (Tabela 3). Os resultados alcançados nesse estudo foram parecidos aos alcançados por Hentz et al. (2014), em que, o SAVI foi o IV designado em seu modelo no processo de estimação da biomassa em uma Floresta Ombrófila Mista no Paraná. Não se verificaram variações sistemáticas após a análise dos resíduos, os gráficos de probabilidade normal se aproximaram de uma linha reta (Figura 2) e a normalidade dos resíduos não foi rejeitada pelo teste de Shapiro-Wilk.

TABELA 3: Propriedades estatísticas da função ajustado para biomassa total.

TABLE 3: Statistical properties of the fitted model.

\begin{tabular}{ccccccc}
\hline Função & Equação & $\mathrm{SQR}$ & $\mathrm{R}^{2}$ & $\mathrm{R}_{\text {aj }}^{2}$ & PRESS & APRESS \\
\hline M1 & Bio $=-1056,7+1428,8 \times$ SAVI & 14596,1 & 0,646 & 0,633 & 0,001 & 0,095 \\
M2 & Bio $=-1588,4+1431,6$ x NDVI & 14691,4 & 0,644 & 0,63 & 0,001 & 0,095 \\
M3 & Bio= $=49,488+589,82 \times$ SR & 15149,7 & 0,633 & 0,619 & 0,001 & 0,096 \\
M4 & Bio $=-975,56-136,19 \times$ EVI & 21590,4 & 0,477 & 0,457 & 0,001 & 0,100 \\
\hline
\end{tabular}

Com o fechamento incompleto das copas das árvores, notou-se a presença de sinais de solo na imagem, propiciando resultados superiores para o $S A V I$, o qual, consequentemente, originou melhores comportamentos dos modelos. Autores como Robinove et al. (1981) salientam que quando não existe o total fechamento do dossel florestal, o sinal espectral mais intenso é do brilho do solo ou sub-bosque. Para Ponzoni e Shimakuburo, (2009), um incremento na taxa de revestimento do solo não aumenta NDVI, devido a sua saturação rápida, porque o índice se estabiliza, mesmo que tivesse verificado um desenvolvimento na densidade do dossel, o IV se torna insensível ao aumento da biomassa. 

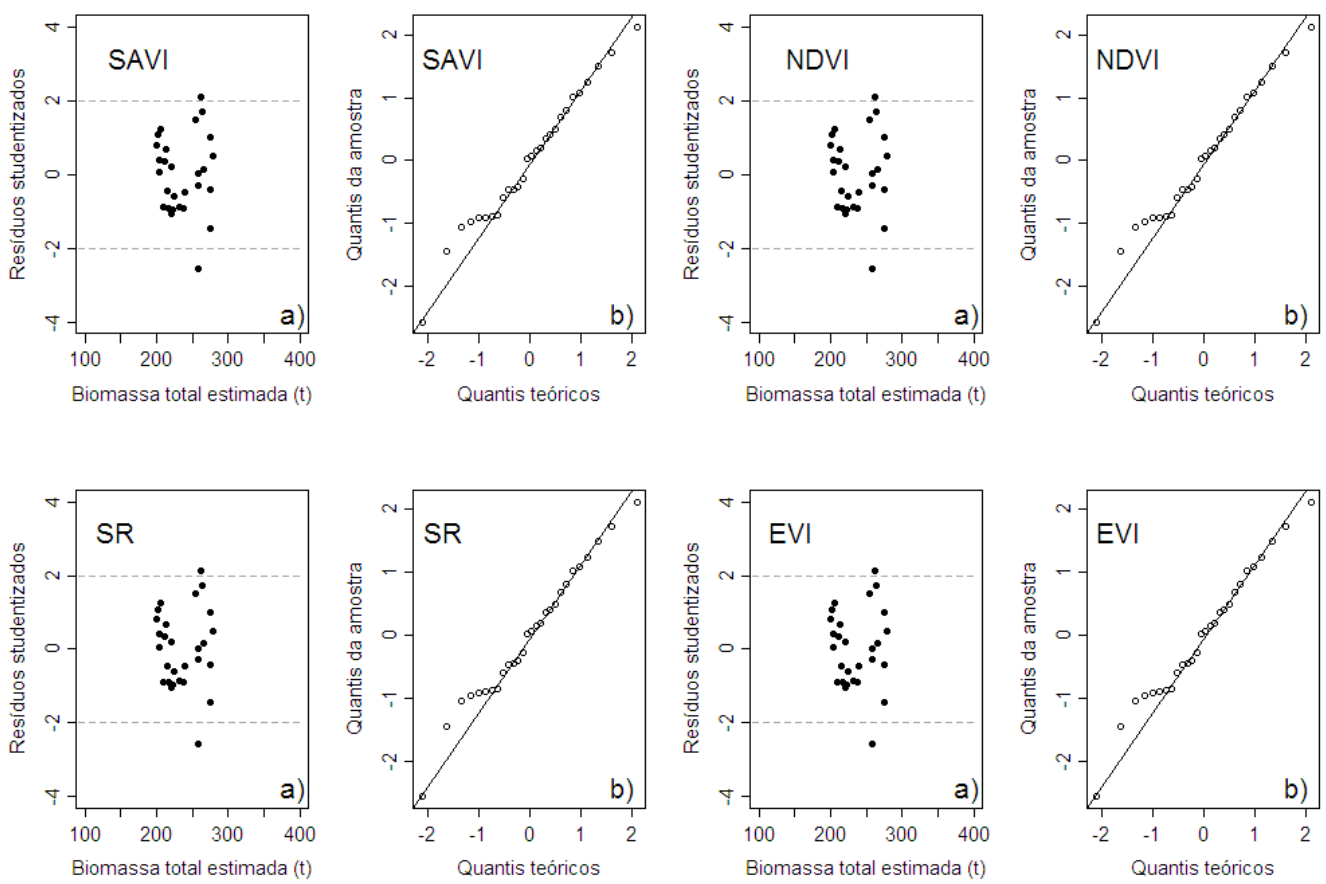

FIGURA 2: Gráficos dos resíduos (a) e de probabilidade normal (b).

FIGURE 2: Studetinzed residuals (a) and normal probability (b) plots.

\section{Mapeamento da Biomassa Total}

Verificou-se uma grande variabilidade entre talhões, supostamente associada à heterogeneidade das idades dos talhões 1, 2, 4 e 7, visto que os mesmos foram plantados 5 meses depois que os talhões 3, 5 e 6 (Figura 3). No grupo inicial, o valor de biomassa total estimada foi menor, provavelmente devido às diferenças de idade dos indivíduos. No entanto, como as árvores do primeiro grupo estão em uma fase de crescimento inicial existe um maior investimento (de fotoassimilados) no desenvolvimento do sistema radicular e no crescimento em altura, e um menor investimento no crescimento em diâmetro. Dentro de cada talhão verifica-se que a variabilidade é menor nos mais velhos e maior nos mais novos, esse efeito pode ser explicado pela variabilidade de nichos dentro de cada talhão, ou seja, quanto maior o espaço de crescimento de cada indivíduo maior o seu crescimento.

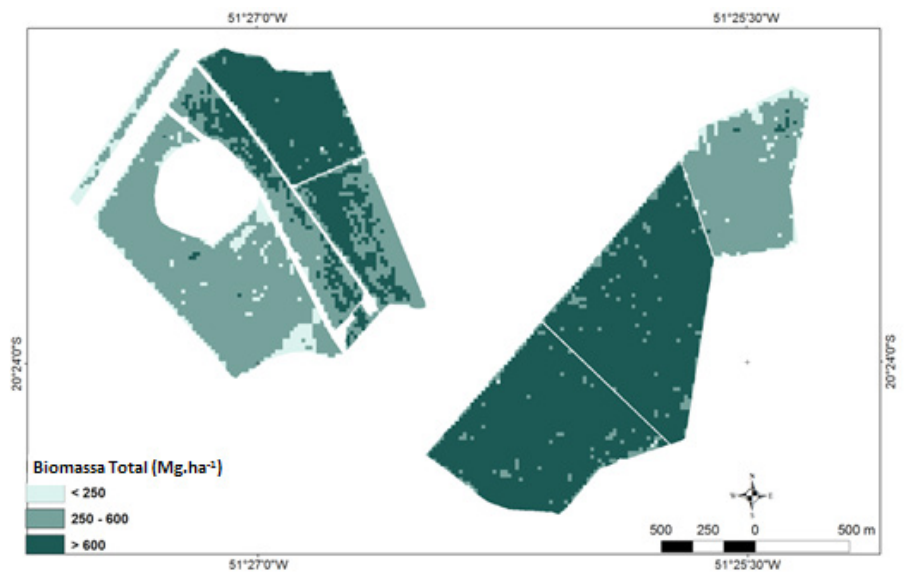

FIGURA 3: Mapa de Biomassa Total para plantios de eucalipto.

FIGURE 3: Maps of Total Biomass for eucalypts plantations. 


\section{CONCLUSÕES}

Neste estudo verificou-se que os dados gerados por meio de imagens de satélite, apresentam uma boa contribuição para a estimativa da biomassa total do Eucalyptus, nomeadamente através da utilização dos índices de vegetação.

A função que apresentou melhor performance na estimativa da biomassa total, foi aquela que utilizou como variável independente o IV-SAVI, apresentando um coeficiente de determinação (R2) de $64,6 \%$, no entanto, sem grande diferença para o NDVI e SR.

Funções desenvolvidas por meio dessas técnicas poderão ser utilizadas em outras regiões que possuam as mesmas espécies, clima e propriedades locais parecidas com as do presente estudo. Esta abordagem poderá ser utilizada como ferramenta de custo relativamente baixo para produzir estimativas de biomassa total em escala local e regional.

Através das estimativas de biomassa total com as funções ajustadas, foi também possível observar que, dentro de uma mesma espécie, o desenvolvimento das mesmas pode ser desuniforme, apresentando variações nos valores estimados de biomassa total.

O mapeamento permite uma análise da biomassa total e o consequente monitoramento do plantio, que pode ser realizado em ambas as séries espaciais e temporais com imagens de satélite Pléiades, visto que as mesmas estão disponíveis em intervalos de tempo curtos e com preços razoáveis.

\section{AGRADECIMENTOS}

Ao Conselho Nacional de Desenvolvimento Científico e Tecnológico e ao Programa Ciência Sem Fronteira (CNPQ) pela concessão da bolsa de estudos para realização do Doutorado Sanduíche junto à Universidade de Évora - Portugal. A imagem Pléiades disponibilizada pela Universidade Estadual Paulista "Júlio de Mesquita Filho" (Unesp - Campus de Ilha Solteira). Pelos dados do inventário florestal cedidos pela empresa Eldorado Brasil.

Este estudo foi ainda cofinanciado pelos fundos do FEDER por meio do Programa Operacional Factores de Competitividade - COMPETE e pelos fundos Nacionais da FCT - Fundação para a Ciência e Tecnologia no âmbito do projeto UID/AGR/00115/2013.

\section{REFERÊNCIAS}

CLUTTER, J. L. et al. Timber management: a quantitative approach. Nova Jersey: John Wiley \& Sons, 1983.

DRAKE, J. B. et al. Estimation of tropical forest structural characteristics using large-footprint lidar. Remote Sensing of Environment, New York, v. 79, n. 2-3, p. 305-319, fev. 2002a.

DRAKE, J. B. et al. Sensitivity of large-footprint lidar to canopy structure and biomass in a neotropical rainforest. Remote Sensing of Environment, New York, v. 81, n. 2-3, p. 378-392, ago. 2002b.

EMBRAPA. Centro Nacional de Pesquisa de Solos. Sistema brasileiro de classificação de solos. Brasília: SPI; CNPS, 1999. 412 p.

ENVIRONMENTAL SYSTEMS RESEARCH INSTITUTE. ArcGIS Desktop: release 10. Redlands: ESRI, 2010.

FEARNSIDE, P. M.; GUIMARÃES, W. M. Carbon uptake by secondary forests in Brazilian Amazonia. Forest Ecology and Management, Amsterdam, v. 80, n. 1-3, p. 35-46, jan. 1996.

GARCIA, C. M. P. et al. Decomposição da palhada de forrageiras em função da adubação nitrogenada após o consórcio com milho e produtividade da soja em sucessão. Bragantia, Campinas, v. 73, n. 2, p. 143-152, 2014.

HIGUCHI, N. et al. Biomassa da parte aérea da vegetação da floresta tropical úmida de terra-firma da Amazônica Brasileira. Acta Amazônica, Manaus, v. 28, n. 2, p. 153-166, jun. 1998.

HENTZ, A. M. K. et al. Técnicas de sensoriamento remoto para estimativa de biomassa em ambientes florestais. Enciclopédia Biosfera, Goiânia, v. 10, n. 18, p. 2810, jul. 2014.

HUETE A. R. A. Soil-Adjusted Vegetation Index (SAVI). Remote Sensing of Environment, New York, 
v. 25 , n. 3, p. 295-309, ago. 1988.

HUETE, A. R.; JUSTICE, C.; VAN LEEUWEN, W. MODIS vegetation index (MOD13): algorithm theoretical basis document, version 2. Tucson: University of Arizona, 1996.

HUETE, A. R. et al. A Comparison of vegetation indices over a global set of TM Images for EOS-MODIS. Remote Sensing of Environment, New York, v. 59, n. 3, p. 440-451, mar. 1997.

INTERGOVERNMENTAL PANEL ON CLIMATE CHANGE. Climate Change: synthesis report. Contribution of working group I, II, and III to the third assessment report of the IPCC. Cambridge: Cambridge University, 2001.

JACKSON, R. D.; HUETE, A. R. Interpreting vegetation indices. Preventive veterinary medicine, Amsterdam, v. 11, n. 3-4, p. 185-200, dez. 1991.

JORDAN, C. F. Derivation of leaf area index from quality of light on the forest floor. Ecology, Washington, v. 50, n. 4, p. 663-666, jul. 1969.

MACIEL, M. N. M. Estimativa de parâmetros estruturais de uma floresta primária na Amazônia Oriental através de dados orbitais. 2002. 144 f. Tese (Doutorado em Ciências Florestais) - Universidade Federal do Paraná, Curitiba, 2002.

MATOS, F. D. A.; KIRCHNER, F. F. Estimativa de biomassa da Floresta Ombrófila Densa de terra firme na Amazônia Central com o satélite IKONOS II. Floresta, Curitiba, v. 38, n. 1, p. 157-171, jan./mar. 2008. MATHEUS, M. T. Sequestro de carbono sob a óptica floresta no Brasil. Revista Trópica - Ciências Agrárias e Biológicas, Chapadinha, v. 6, n. 1, p. 104-116, jan. 2012.

MELLO, S. L. M.; GONÇALVES, J. L. M. Equações para estimar a biomassa da parte aérea e do sistema radicular em povoamentos de Eucalyptus grandis em sítios com produtividades distintas. Revista Árvore, Viçosa, MG, v. 32, n. 1, p. 101-111, jan./fev. 2008.

MYERS, R. H. Classical and modern regression with applications. Boston: Duxbury, 1986.

NUNES, S. do S. S. Estimativa de biomassa e carbono e indicadores para restauração de florestas secundárias em Paragominhas, Pará. 2011. 127 f. Dissertação (Mestrado em Recursos Florestais) Escola Superior de Agricultura, Universidade de São Paulo, Piracicaba, 2011.

PONZONI, F. J.; SHIMABUKURU, Y. E. Sensoriamento remoto no estudo da vegetação. São José dos Campos: A. Silva Vieira, 2009. 150 p.

R DEVELOPMENT CORE TEAM. R: a language and environment for statistical computing. Vienna: R Foundation for Statistical Computing, 2012.

RAUSTE, J.; HÄME, T. Radar-based forest biomass estimation. International Journal of Remote Sensing, London, v. 15, n. 14, p. 2797-2808, dez. 1994.

RENNER, R. M. Sequestro de carbono e a viabilização de novos reflorestamentos no Brasil. 2004. 147 f. Dissertação (Mestrado em Engenharia Florestal) - Universidade Federal do Paraná, Curitiba, 2004. ROBINOVE, C. J. et al. Arid land monitoring using Landsat Albedo Difference Images. Remote Sensing of Environment, New York, v. 11, p. 133-156, 1981.

ROSENQVIST, A. et al. A review of remote sensing technology in support of the Kyoto protocol. Environmental Science \& Policy, Amsterdam, v. 6, p. 441-455, fev. 2003.

ROUSE, J. W. et al. Monitoring vegetation systems in the Great Plains with ERTS. In: ERTS-1 SYMPOSIUM, 3., 1974, Washington. Proceedings... Washington: NASA Goddard; NASA SP-351, 1974. p. 309-317.

SANQUETTA, C. R. Métodos de determinação de biomassa florestal. In: SANQUETTA, C. R. et al. (Ed.). As florestas e o carbono. Curitiba: [s. n.], 2002. p. 119-140.

SHAPIRO, S. S.; WILK, M. B.; CHEN, H. J. A comparative study of various tests of normality. Journal of the American Statistical Association, Washington, v. 63, n. 324, p. 1343-1372, dez. 1968.

SOMOGYI, Z. et al. Indirect methods of large forest biomass estimation. European Journal of Forest Research, Berlin, v. 126, n. 2, p. 197-207, mai. 2006.

SOUSA, A. M. O. et al. Biomass estimation with high resolution satellite images: a case study of Quercus rotundifolia. ISPRS Journal of Photogrammetry and Remote Sensing, Amsterdam, v. 101, p. 69-79, mar. 2015.

VISMARA, E. S. Mensuração da biomassa e construção de modelos para construção de equações de biomassa. 2009. 103 f. Dissertação (Mestrado em Recursos Florestais) - Universidade de São Paulo, Piracicaba, 2009. 
WATZLAWICK, L. F. et al. O papel do sensoriamento remoto nos estudos de carbono. In: SANQUETTA, C. R. et al. (Ed.). As florestas e o carbono. Curitiba: Brasil, 2002. 265 p.

WATZLAWICK, L. F.; KIRCHNER, F. F. Estimativa de biomassa e carbono utilizando imagens de satélite de alta resolução: In: SANQUETTA, C. R.; BALBINOT, R.; ZILIOTTO, M. A. A. (Ed.). Fixação de carbono: atualidades, projetos e pesquisas. Curitiba: [s. n.], 2004. p. 133-152.

WATZLAWICK, L. F.; KIRCHNER, F. F.; SANQUETTA, C. R. Estimativa de biomassa e carbono em floresta com araucária utilizando imagens do satélite Ikonos II. Ciência Florestal, Santa Maria, v. 19, n. 2, p. 169-181, abr./jun. 2009.

WATZLAWICK, L. F.; KOEHLER, H. S.; KIRCHNER, F. F. Estimativa de biomassa e carbono em plantios de Pinus taeda L. utilizando imagens do satélite IKONOS II. Ciência e Natura, Santa Maria, v. 28, n. 1, p. 45-60, jan./jul. 2006.

YU, C. M. Sequestro florestal de carbono no Brasil: dimensões políticas socioeconômicas e ecológicas. São Paulo: Annablume; IEB, 2004. 280 p. 